\title{
Functional Polymers Through Mechanochemistry
}

\author{
Stephen Schrett| ${ }^{\star a}$, Diederik W. R. Balkenende ${ }^{\text {ab }}$, Céline Calvino ${ }^{a}$, Marc Karmana, Anna Lavrenova ${ }^{a}$, \\ Laura N. Neumann ${ }^{\mathrm{a}}$, Yoshimitsu Sagara ${ }^{\mathrm{ac}}$, Ester Verde-Sestoad, Michela di Giannantonio ${ }^{\mathrm{e}}$, Yoan C. \\ Simon ${ }^{\ddagger}$, Katharina M. Fromme, Marco Lattuadae, and Christoph Weder ${ }^{\star a}$
}

\begin{abstract}
While coupling mechanical and chemical processes is widespread in living organisms, the idea to harness the mechanically induced dissociation of weak covalent and non-covalent bonds to create artificial materials that respond to mechanical stimulation has only recently gained attention. Here we summarize our activities that mainly revolve around the exploitation of non-covalent interactions in (supramolecular) polymeric materials with the goal to translate mechanical stresses into useful, pre-defined events. Focusing on mechanochromic polymers that alter their optical absorption or fluorescence properties, several new operating principles, mechanosensitive entities, and materials systems were developed. Such materials are expected to be useful for technical applications that range from the detection of very small forces in biological systems to the monitoring of degradation processes and damage in coatings and structural objects.
\end{abstract}

Keywords: Mechanochromic $\cdot$ Mechanophore $\cdot$ Mechanoresponsive $\cdot$ Polymer $\cdot$ Stimuli-responsive polymers

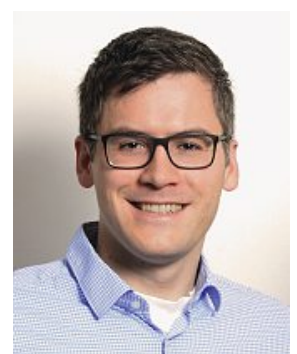

Dr. Stephen Schrettl studied Chemistry at Freie Universität Berlin and received his $\mathrm{PhD}$ in Materials Science from Ecole Polytechnique Fédérale de Lausanne working with Prof. Holger Frauenrath. Following postdoctoral research first at EPFL and then with Prof. Christoph Weder at the Adolphe Merkle Institute (AMI) of the University of Fribourg, he took up his current position as Group Leader at the AMI. His research interests include the structure-property relationship of supramolecular polymers, the development of bio-inspired materials, as well as the preparation of responsive polymers and composites.

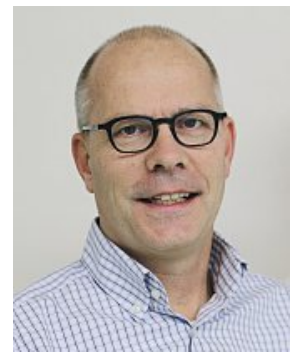

Prof. Dr. Christoph Weder is Director of the Adolphe Merkle Institute (AMI) at the University of Fribourg and leads the Swiss National Center of Competence in Research Bio-Inspired Materials. Chris was educated in Chemistry and Polymer Science at ETH Zurich and held positions at MIT, ETH Zurich, and Case Western Reserve University, before joining the AMI as Professor for Polymer Chemistry and Materials in 2009. His research focuses on the investigation and application of bio-inspired stimuli-responsive polymers and nanomaterials. Chris is a member of the Swiss Academy of Engineering Sciences, a fellow of the American Chemical Society's Division of Polymer Chemistry, associate editor of ACS Macro Letters, and coeditor of the RSC Book Series Polymer Chemistry.

\section{Introduction}

Many materials systems found in living organisms have the ability to adapt their properties to specific situations, often in response to external cues. ${ }^{[1]}$ Among the various transduction schemes, mechanochemical processes, which connect mechanical to chemical events, are omnipresent in nature. ${ }^{[2]}$ For example, the bioluminescence of dinoflagellates is triggered by force-induced deformations of the cell membranes, ${ }^{[3]}$ and force transduction to mechanosensitive ion-channels is the basis for the sense of touch. ${ }^{[4]}$ The idea to create synthetic materials that are inspired by such principles and which respond to mechanical stimulation with a useful property change has gained much attention over the last two decades, although such engineered materials rely, of course, on much simpler designs.

It has long been known that in synthetic polymeric materials, stresses generally lead to an unspecific, irreversible scission of the constituting polymer chains.[5] Indeed, the observation that the molecular weight of poly(styrene) decreases in response to high shear forces dates back to Staudinger. ${ }^{[6]}$ Experimental and theoretical investigations have since established a thorough understanding of the mechanically induced changes that occur in polymeric materials on the molecular level.[7] Polymers initially respond to tensile deformation with the extension of individual macromolecules, which stretch and uncoil, before homolytic or heterolytic cleavage of covalent bonds occurs. ${ }^{[8]}$ The idea to exploit this mechanism for the design of polymeric materials that translate mechanical stresses into useful responses and functions relies on the incorporation of 'weak links', also referred to as mechanophores..$^{[9,10]}$ Motifs such as spiropyran-, ${ }^{[11]}$ dioxetane-, ${ }^{[12]}$ epoxide--, ${ }^{[13]}$ and azo-moieties, ${ }^{[14]}$ have been explored in this context and were shown to undergo mechanically induced chemical

\footnotetext{
${ }^{*}$ Correspondence: Prof. C. Wedera, Dr. S. Schrettla

E-mail: christoph.weder@unifr.ch, stephen.schrettl@unifr.ch

adolphe Merkle Institute, University of Fribourg, Chemin des Verdiers 4, CH-1700 Fribourg

${ }^{b}$ Department of Materials Science and Engineering, University of California Berkeley, 130D Hearst Memorial Mining Building, Berkeley CA 94720-1760, USA

${ }^{\mathrm{c}}$ Research Institute for Electronic Science, Hokkaido University, N20, W10, Kita-Ku, Sapporo 001-0020, Japan

${ }^{d}$ Centro Joxe Mari Korta, Avenida de Tolosa, 72, 20018 Donostia-San Sebastián, Spain

eDepartment of Chemistry, University of Fribourg, Chemin du Musée 9, $\mathrm{CH}-1700$ Fribourg

fSchool of Polymer Science and Engineering, The University of Southern Mississippi, 118 College Dr., Box 5050, Hattiesburg, MS 39406, USA
} 
changes that lead to mechanochromic behavior, mechanically induced chemiluminescence, and other functions. With the notion that non-covalent interactions play an important role in (responsive) biological materials. ${ }^{[15,16]}$ we embarked on the exploration of non-covalent interactions to access new (supramolecular) polymers with useful stimuli-responsive functionalities. ${ }^{[17]}$

\section{From Mechanically-triggered Metal Release to Displacement-activated Mechanophores}

Early on, metal-ligand coordination bonds have been recognized as motifs that can be (reversibly) dissociated when exposed to mechanical force; examples include Pd-phosphane complexes, ${ }^{[18]}$ $\mathrm{Ag}-$, $\mathrm{Ru}-$, or $\mathrm{Cu}$-carbene complexes, ${ }^{[19-21]}$ as well as $\mathrm{Zn}^{2+}-, \mathrm{Eu}^{3+}-$, $\mathrm{Tb}^{3+}$, , or $\mathrm{La}^{3+}$-based metallosupramolecular polymers (MSPs). ${ }^{[22-25]}$ For instance, we have shown that metallopolymer networks assembled from a telechelic poly(ethylene-co-butylene) terminated with 2,6-bis(1'-methylbenzimidazolyl)pyridine ligands and $\mathrm{Eu}\left(\mathrm{ClO}_{4}\right)_{3}$ exhibit remarkable fluorescence intensity changes upon mechanical stimulation, as the red fluorescing, dynamic Mebip-Eu ${ }^{3+}$ complexes function as built-in optical probes that allow for a monitoring of the extent of (dis)assembly. ${ }^{[22,26]}$ The relative weakness of such non-covalent interactions, their tunable dynamic character, and their reversibility - which contrasts the behavior of most covalent mechanophores - render them ideal to serve as pre-defined weak links in polymeric materials. Similar MSPs are also attractive candidates for other sensing systems, as they can be processed into coatings and self-supporting films, whose luminescence changes in response to different chemical analytes. ${ }^{[27]}$

With regards to more strongly bound metal-ligand complexes, we identified ferrocene $(\mathrm{Fc})$ as a potentially interesting mechanically responsive motif, based on the notion that the dissociation enthalpy of the Fc motif is lower than that of $\mathrm{C}-\mathrm{C}, \mathrm{C}-\mathrm{O}$, and $\mathrm{C}-\mathrm{N}$ bonds present in 'typical' polymer backbones. ${ }^{[28]}$ Thus, polyurethanes (PUs) with multiple, randomly distributed Fc units (Fc-PU) in their backbone, and poly(methyl acrylate)s (PMA) containing a single Fc unit in the center of each macromolecule (Fc-PMA) were prepared (Fig. 1a). The response of the Fc-containing and the mechanophore-free reference polymers to mechanical stimulation was studied by ultrasonication of solutions. The reduction of their molecular weight was monitored by size exclusion chromatography (SEC) as a function of sonication time (Fig. 1b). The evaluation of the chain scission kinetics clearly confirmed the preferred scission of the Fc-mechanophore. The release of iron ions from mechanically cleaved Fc units was demonstrated by addition of potassium thiocyanate and the rapid formation of colored thiocyanatoiron complexes (Fig. 1c). Accordingly, Fc-type complexes are viable mechanophores and the concept should be readily applicable to other metallocene-type complexes with potential applications spanning from colorimetric sensing to catalysis. The versatility of the approach was recently confirmed by Tang, Craig, and coworkers, who not only integrated the Fc motifs in other polymers, but also demonstrated that the mechanically induced $\mathrm{Fc}$ cleavage occurs through a predominately heterolytic mechanism that involves the initial dissociation into $[\mathrm{CpFe}]^{+}$and $\mathrm{Cp}^{-}$. ${ }^{[29]}$

To detect particularly weak forces, a fundamentally new mechanochemical transduction principle was conceived, which is based on exploiting the molecular-shuttle function of rotaxanes. The principle relies on the idea that the positional rearrangement upon reversible displacement of two mechanically interlocked molecules can be designed to elicit an optical signal. As a first embodiment, a benzothiadiazole fluorophore macrocycle was threaded onto a dumbbell-shaped molecule containing a naphthalene bisimide as a fluorescence quencher (Fig. 2). ${ }^{[30]}$ The rotaxane, whose fluorescence is quenched in the idle state, was covalently incorporated in the backbone of a thermoplastic polyurethane elastomer. This material does not show any fluorescence, but the characteristic benzothiadiazole fluorescence is gradually turned on upon defor-
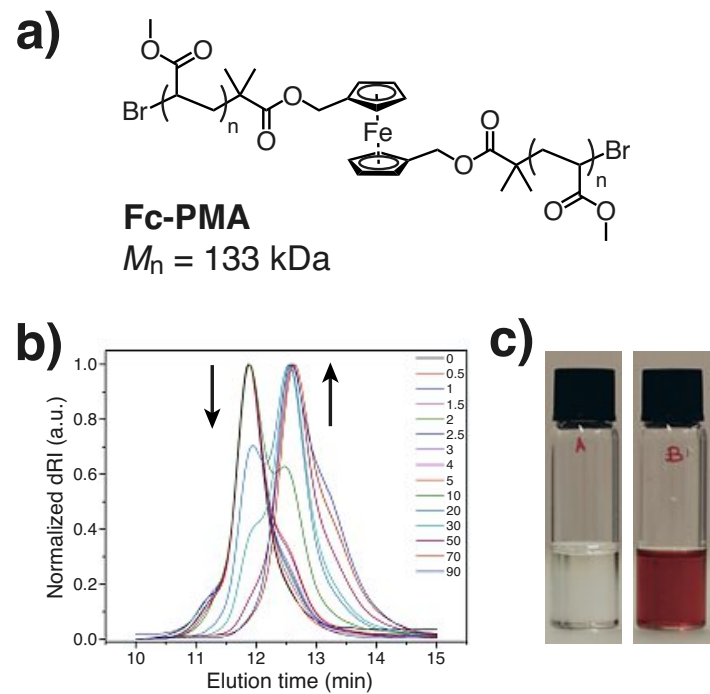

Fig. 1. (a) Poly(methyl acrylate) with a single ferrocene unit in the center (Fc-PMA). (b) Size exclusion chromatography traces as a function of ultrasonication time of a Fc-PMA solution show a reduction of molecular weight. (c) Potassium thiocyanate was added to solutions of Fc-PMA before (left) and after 90 min of sonication (right). The rapid formation of the red Fe-thiocyanate complex demonstrated the release of iron ions. Adapted with permission from ref. [28b]; Copyright 2018 John Wiley and Sons.

mation (Fig. 2c), due to the separation of the mechanically interlocked fluorophore/quencher pair. Our investigations showed that the process can only be activated by mechanical force (and not heat or light), that the mechanism is very efficient and reversible, and that the optical signal correlates with the extent of deformation. The mechanism fundamentally differs from the chemical activation exploited in other mechanophores, as it relies on a mechanical trapping of an entropically unfavorable configuration. This principle should be applicable to other interlocked architectures and is useful for the detection of very small forces that are otherwise difficult to assess, e.g. in biological systems.

In addition to the above non-covalent mechanophores, several covalent mechanophores were developed and studied. For example, diaryldibenzofuranone, a scissile dynamic covalent mechanophore, was covalently bound to the surface of cellulose nanocrystals (CNCs) and the particles were used to create a nanocomposite with a healable polymer containing the same dynamic motif. The formation of reversible covalent bonds between the modified $\mathrm{CNCs}$ and the matrix polymer imparted the composites not only with mechanochromic behavior, but also with the ability to heal.[31] Moreover, the mechanochromic response of dithiomaleimide- as well as benzoxazole-containing polymers were explored. For example, the fluorescent dithiomaleimide-motif was placed in the center of either PMA or poly( $\varepsilon$-caprolactone) (PCL) and acted as a mechanophore that was selectively cleaved when exposed to ultrasonication in solution, causing a corresponding fluorescence intensity decrease. ${ }^{\left[{ }^{32}\right]}$ Similarly, an aliphatic 2-(2'-hydroxyphenyl) benzoxazole ester was employed as a mechanofluorophore;[33] here, an excited-state intramolecular proton transfer is enabled upon sonochemical cleavage of the ester moieties, which in turn causes a marked change of the emission color. ${ }^{[34]}$

\section{Mechanoresponsive Luminescent (Supramolecular) Polymers}

The above examples reflect that there is tremendous interest in materials that display mechanoresponsive luminescence (MRL) behavior, which is in part motivated by academic curiosity and in part by the goal to provide materials that enable the examination of structural integrity or detection of tampering. ${ }^{[35]} \mathrm{We}$ first reported an MRL effect in blends of different polymers and small 

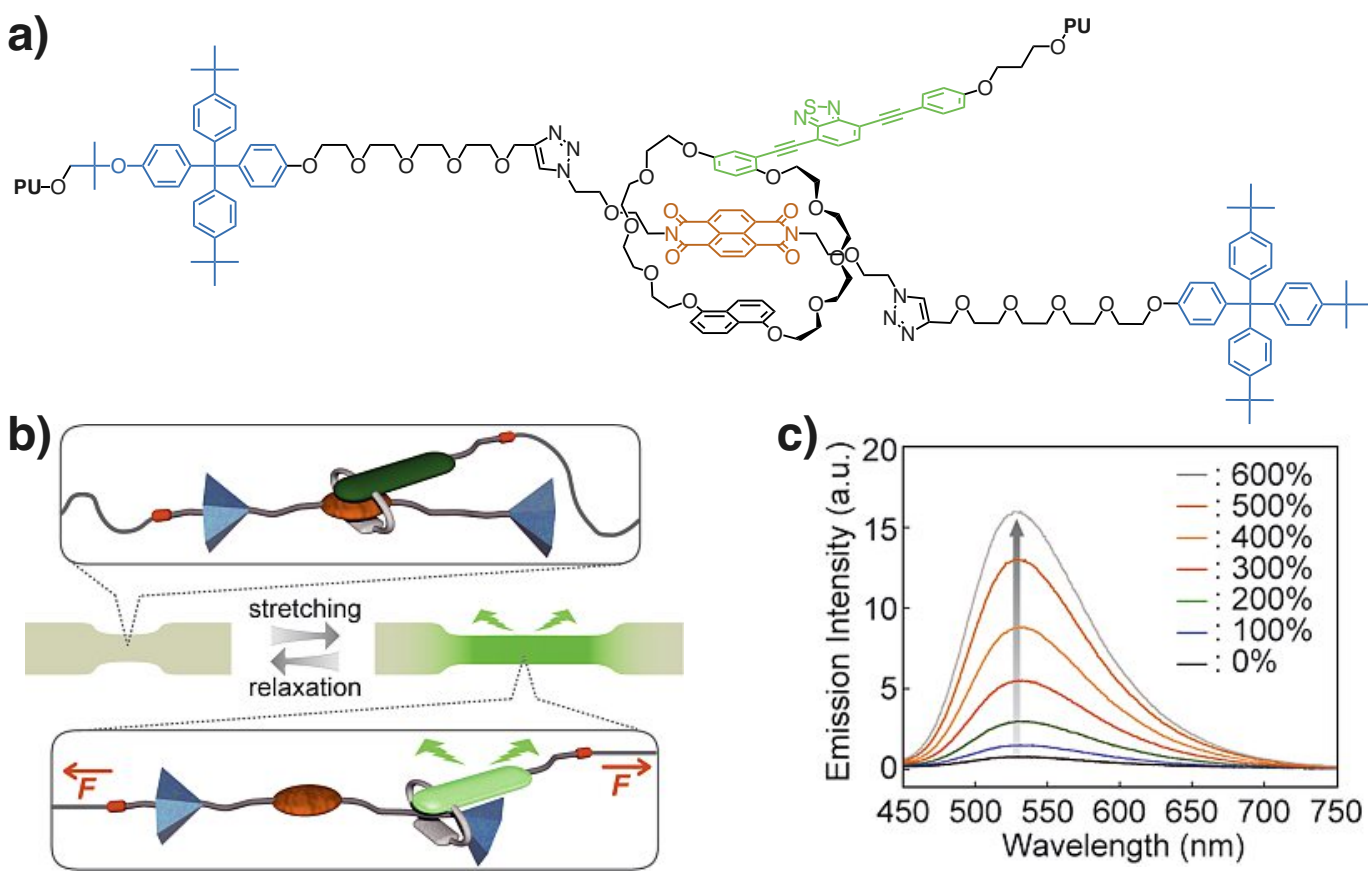

Fig. 2. (a) Chemical structure of the rotaxane-based mechanoluminophore that was incorporated in a polyurethane (PU). (b) Schematic depiction of the operating principle of a mechanically interlocked mechanophore with a force-induced displacement of a quencher (brown) from a fluorophore (green). (c) Fluorescence emission spectra as a function of applied strain for a film made from the rotaxane containing PU. (d) Photographs of samples of the PU film taken before, during, and after uniaxial tensile deformation show the fluorescence response. Images in the top and bottom were taken under illumination with $365 \mathrm{~nm}$ UV light and ambient light, respectively. Adapted with permission from ref. [30] [https:// pubs.acs.org/doi/pdf/10.1021/ jacs.7b12405]; Copyright 2018 American Chemical Society.

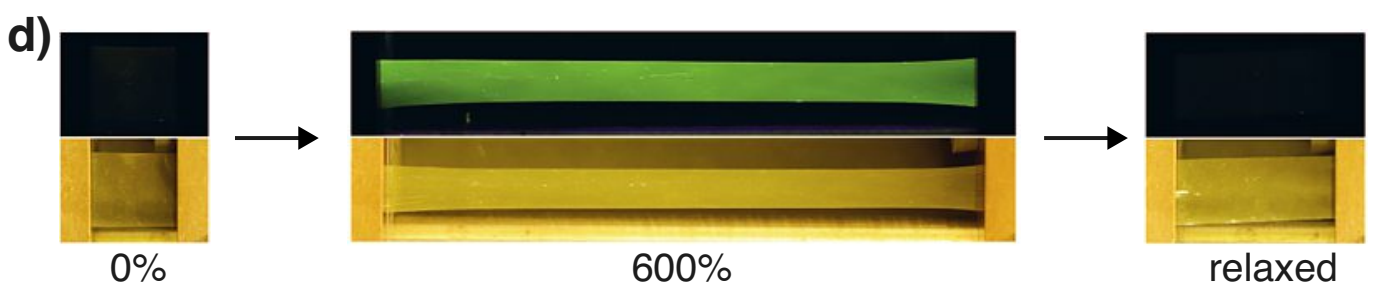

amounts of photoluminescent cyano-substituted oligo(phenylene vinylene) (cyano-OPV) dyes over 15 years ago.[36] This approach requires both the formation of nanometer-sized dye aggregates in a given matrix, as well as their disruption through an efficient stress-transfer upon bulk mechanical deformation of samples. ${ }^{[37,38]}$ This mechanism, which can also be exploited with aggregachromic molecules, ${ }^{[39]}$ works particularly well in semicrystalline host polymers, because of nucleation effects as well as particular stress transfer mechanisms. For example, we recently rendered polyamide 12 mechanochromic by blending this material with 0.15 $1 \mathrm{wt} \%$ of a cyano-OPV. ${ }^{[40]}$ Blends with a low dye concentration $(0.15 \%)$ displayed the green monomer fluorescence that is characteristic of molecularly dissolved molecules, whereas aggregation of the dye at higher concentrations led to red excimer emission. The deformation of samples with a dye content of $0.25 \mathrm{wt} \%$ caused a pronounced fluorescence color change from an excimer- to a monomer-rich emission. While this approach has been realized for select combinations of dyes and matrix polymers, recent results suggest that it can be extended to a much broader palette of polymers by employing a telechelic poly(ethylene-co-butylene) that features the excimer-forming cyano-OPV fluorophores at the two termini. ${ }^{[41]}$ We showed that the incorporation of $0.1-2 \mathrm{wt} \%$ of this macromolecule bestowed different types of polymers with MRL behavior (Fig. 3). Thus, blends of poly(styrene- $b$-butadiene- $b$-styrene), poly(isoprene), or poly(caprolactone) with the cyano-OPV terminated telechelic all displayed fluorescence color changes in response to bulk mechanical deformation. The results suggest that the stress-transfer to the dye aggregates can be significantly improved by coupling the chromophores to a telechelic 'carrier', presumably because the latter entangles with the matrix polymer.

About a decade ago, it was discovered that certain organic and organometallic dyes, including the above-mentioned cyanoOPVs, can display MRL behavior on their own, i.e. without being embedded in a polymer. ${ }^{[43-45]}$ Such dyes display distinct emissive colors depending on their assembly, with some cyano-OPV deriva- tives adopting up to five different assembly and emissive states. ${ }^{[46]}$ Conversion between the latter is possible by thermal or mechanical treatment, ${ }^{[46,47]}$ which renders these dyes a priori useful for the creation of stimuli-responsive luminescent materials. In the meantime, several hundred dyes with MRL behavior have been reported, ${ }^{[35]}$ but due to their low molecular weight, these compounds don't exhibit any mechanical properties to speak of. To mitigate this, we devised materials that combine the concept of supramolecular polymerization and the responsive behavior of MRL compounds. In a first example of this approach, self-complementary hydrogenbonding ureido-4-pyrimidinone (UPy) groups were employed to self-assemble an excimer-forming cyano-OPV derivative into a supramolecular polymer (Fig. 3c-e). ${ }^{[42]}$ This material displays the characteristics of a supramolecular polymer glass, with a storage modulus of $c a .1 .5 \mathrm{GPa}$ and a glass transition temperature of around $90{ }^{\circ} \mathrm{C}$. The material can be melt-processed into self-supporting films, fibers, and 3-D objects, which exhibit both MRL as well as thermoresponsive luminescent behavior. Moreover, it was demonstrated that patterning of this material with nanometersized optical and topographical features can be achieved by local thermal or mechanical stimulation with thermal scanning probe lithography, providing a direct path towards information storage systems. ${ }^{[48]}$ This conceptual approach should be applicable to other MRL molecules as well as alternative binding motifs, and should therefore be broadly exploitable for the creation of materials with tailored stimuli-responsive luminescent behaviors.

\section{Self-reporting, Microcapsule-containing Polymer Materials}

Another versatile approach towards polymeric materials that exhibit a chromic response to mechanical stimulation is the use of dye-filled microcapsules. ${ }^{[49]}$ The principal advantage of this concept is that such microcapsules can be incorporated into any kind of polymer, independent of the employed dye, the solvent, or the chemical characteristics of the capsule. Deformation of the 
a)

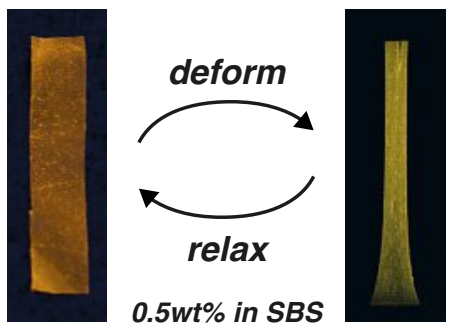

b)

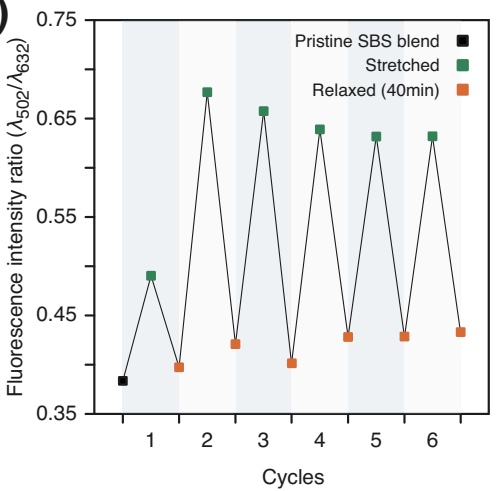

c)

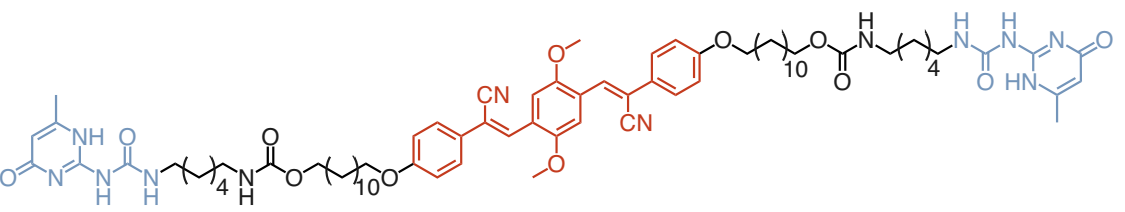

d)

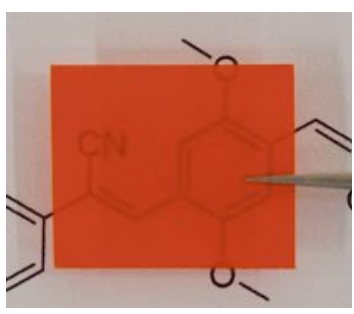

e)

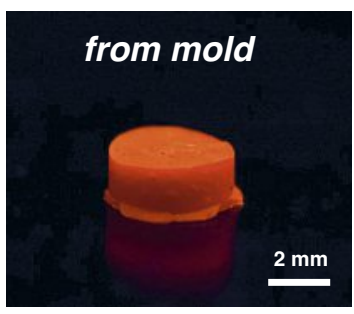

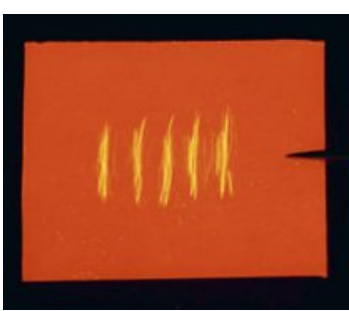

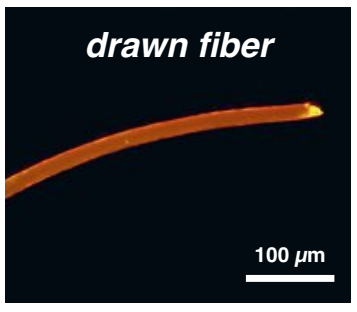

Fig. 3. (a) Photographs of a poly(styrene-b-butadiene- $b$-styrene) (SBS) blend with 0.5 wt $\%$ of the aggregachromic macromolecule taken before (left) and upon (right) stretching the film to $730 \%$ strain. (b) Plot of the monomer-to-excimer emission intensity ratios determined in repeated deformation cycles (730\% strain and relaxation to 0\% strain). Adapted with permission from ref. [41]; Copyright 2018 John Wiley and Sons. (c) Chemical structure of the excimer-forming cyano-OPV derivative with hydrogen-bonding UPy groups. (d) Photograph of a film of the UPy-functionalized cyanoOPV and response upon scratching the surface, as indicated by a change of the emission color from red to yellow. (e) Photographs of a cylinder as well as a fluorescence microscopy image of a fiber made from the UPy-functionalized cyano-OPV. Adapted with permission from ref. [42]; Copyright 2017 American Chemical Society. All images displaying fluorescence were taken under illumination with $365 \mathrm{~nm}$ UV light.

bulk composite damages the embedded microcapsules, which in turn release their cargo and the desired mechanochromic effect. To maximize the observed visual contrast, the dye system should ideally be chemically or physically activated upon release, and, depending on the employed type of dyes, this conceptually simple approach can be readily used to achieve versatile mechanochromic responses. ${ }^{[50-52]}$ In one embodiment of this approach, microcapsules filled with charge-transfer complex precursors were used to visualize polymer deformation. ${ }^{[53]}$ Thus, microcapsules consisting of a poly(urea-formaldehyde) shell, toluene as the solvent, and either chloranil as acceptor or hexamethylbenzene as donor were prepared by emulsion polymerization (Fig. 4a). The microcapsule pairs were embedded in a poly(dimethylsiloxane) (PDMS) matrix by dispersing them in the monomer and subsequently curing the material. The microcapsules ruptured when the material was placed under mechanical load, the donor/acceptor pair was released into the polymeric matrix where they formed a red-colored charge-transfer complex (Fig. 4b).

The versatile nature of this approach was demonstrated by combining the mechanically controlled release of a cargo with the concept of aggregation-induced excimer-formation of cyanoOPV dyes (Fig. 4c). ${ }^{[54]}$ Thus, poly(urea-formaldehyde) microcapsules were filled with a cyano-OPV solution and embedded in PDMS, an epoxy resin, as well as a styrene-butadiene rubber. In all cases, capsule rupture upon deformation of the composites furnished a pronounced fluorescence change due to excimer formation in the damaged area. Detailed investigations with the silicone composites demonstrated that impact, incision, compression, and tensile deformation all triggered the chromic response. Moreover, the emission from unopened capsules was found to serve as a built-in reference, rendering the response a ratiometric signal and the materials self-calibrating (Fig. 4d,e). In this sense, the evaluation of both spectroscopic data as well as RGB color analyses of pictures taken under UV light allowed for a quantification of the mechanical damage and an assessment of the structural integrity of the composite materials.

\section{Conclusions}

The research outcomes summarized here demonstrate that non-covalent interactions are an attractive design element for mechanoresponsive moieties. Emulating, at least in a conceptual manner, transduction principles found in nature, the responses of materials containing such motifs are generally reversible, dynamic, and readily tunable and several novel functions were demonstrated. As such, these results go significantly beyond the state of the art that relies mostly on the irreversible dissociation of covalent bonds ${ }^{[5]}$ and provide a broad basis for the design of novel materials that translate mechanical stimuli into useful functions. Continued development of novel motifs and detailed investigations of the response of such non-covalent interactions to mechanical stimuli will further increase the understanding of the molecular mechanisms, which in turn is expected to facilitate the design of materials that respond in distinct ways to multiple different stimuli ${ }^{[55]}$ and help to bridge the performance gap between artificial and biological materials.

\section{Acknowledgements}

The authors gratefully acknowledge financial support through the National Center of Competence in Research Bio-Inspired Materials, a research instrument of the Swiss National Science Foundation, the Adolphe Merkle Foundation, and from the European Research Council under the European Union's Seventh Framework Programme (FP7/20072013)/ERC grant agreement no. AdG 291490-MERESPO.

Received: November 21, 2018

[1] L. Montero de Espinosa, W. Meesorn, D. Moatsou, C. Weder, Chem. Rev. 2017, 117, 12851, doi: 10.1021/acs.chemrev.7b00168

[2] P. G. Gillespie, R. G. Walker, Nature 2001, 413, 194, doi: 10.1038/35093011.

[3] S. K. Mallipattu, M. A. Haidekker, P. von Dassow, M. I. Latz, J. A. Frangos, J. Comp. Physiol. A 2002, 188, 409, doi: 10.1007/s00359-002-0315-9.

[4] M. Chalfie, Nat. Rev. Mol. Cell. Biol. 2009, 10, 44, doi: 10.1113/jphysiol.1989.sp017878.

[5] M. M. Caruso, D. A. Davis, Q. Shen, S. A. Odom, N. R. Sottos, S. R. White, J. S. Moore, Chem. Rev. 2009, 109, 5755, doi: 10.1021/cr9001353. 
a)
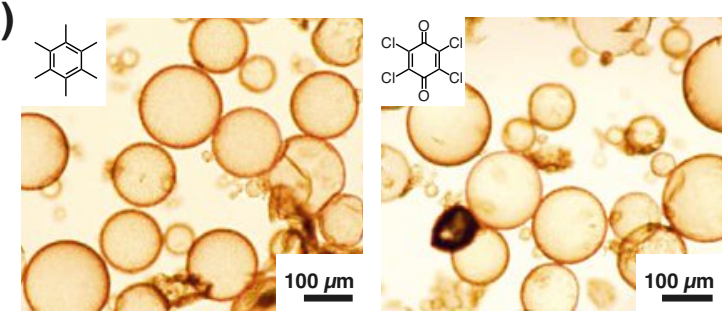

b)

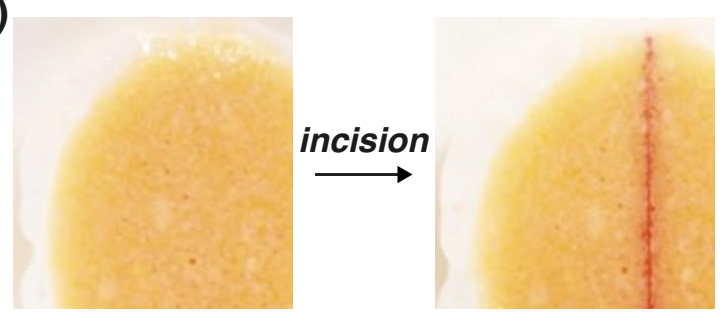

c)
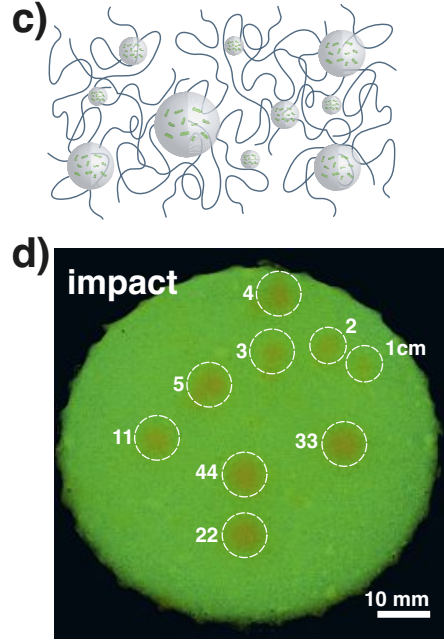

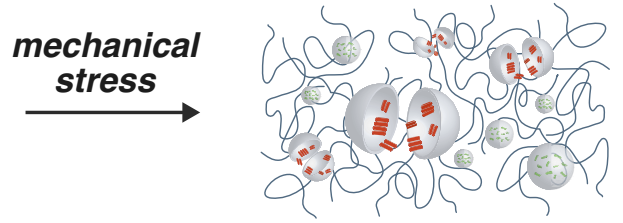

e)

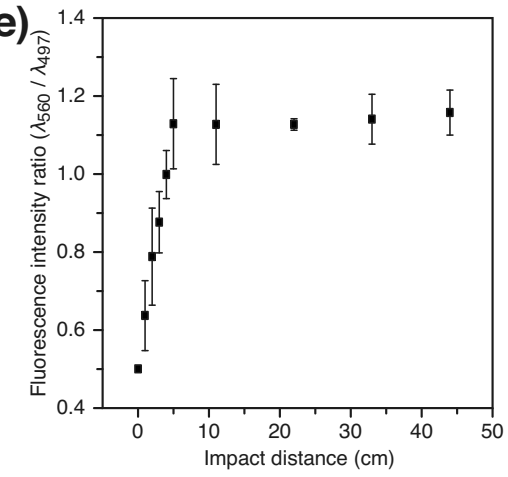

Fig. 4. (a) Microscopy images of poly(urea-formaldehyde) microcapsules with hexamethylbenzene as donor (left) and chloranil as acceptor (right). (b) Photographs of a sample of a poly(dimethylsiloxane) composite containing 10wt\% of the capsules before (left) and after (right) incision. Adapted with permission from ref. [53]; Copyright 2015 American Chemical Society. (c) Schematic depiction of composites featuring microcapsules that contain excimer-form cyano-OPV dyes. (d) Photograph of a sample after exposure to the impact of a missile from distances between 1 and $44 \mathrm{~cm}$ (illumination by $365 \mathrm{~nm}$ UV light). (e) Plot of the excimer-to-monomer emission intensity ratio as a function of impact distance. Adapted with permission from ref. [54]; Copyright 2018 John Wiley and Sons.

[6] H. Staudinger, W. Heuer, Ber. dtsch. Chem. Ges. A/B 1934, 67, 1159, doi: 10.1002/cber.19340670708.

[7] M. K. Beyer, H. Clausen-Schaumann, Chem. Rev. 2005, 105, 2921, doi: 10.1021/cr030697h

[8] R. J. Young, P. A. Lovell, 'Introduction to Polymers, Third Edition', CRC Press, Boca Raton, US, 2011.

[9] A. L. Black, J. M. Lenhardt, S. L. Craig, J. Mater. Chem. 2011, 21, 1655, doi: $10.1039 /$ C0JM02636K.

[10] J. Li, C. Nagamani, J. S. Moore, Acc. Chem. Res. 2015, 48, 2181, doi: 10.1021/acs.accounts.5b00184.

[11] D. A. Davis, A. Hamilton, J. Yang, L. D. Cremar, D. Van Gough, S. L. Potisek, M. T. Ong, P. V. Braun, T. J. Martínez, S. R. White, J. S. Moore, N. R. Sottos, Nature 2009, 459, 68, doi: 10.1038/nature07970.

[12] Y. Chen, A. J. H. Spiering, S. Karthikeyan, G. W. M. Peters, E. W. Meijer, R. P. Sijbesma, Nat. Chem. 2012, 4, 559, doi: 10.1038/nchem.1358.

[13] H. M. Klukovich, Z. S. Kean, A. L. B. Ramirez, J. M. Lenhardt, J. Lin, X. Hu, S. L. Craig, J. Am. Chem. Soc. 2012, 134, 9577, doi: 10.1021/ja302996n.

[14] K. L. Berkowski, S. L. Potisek, C. R. Hickenboth, J. S. Moore, Macromolecules 2005, 38, 8975, doi: 10.1021/ma051394n.

[15] P. Lavalle, F. Boulmedais, P. Schaaf, L. Jierry, Langmuir 2016, 32, 7265, doi: 10.1021/acs.langmuir.6b01768.

[16] M. J. Buehler, Nano Today 2010, 5, 379, DOI: 10.1016/j.nantod.2010.08.001.

[17] C. Calvino, L. Neumann, C. Weder, S. Schrettl, J. Polym. Sci. Part A: Polym. Chem. 2017, 55, 640, doi: 10.1002/pola.28445.

[18] J. M. J. Paulusse, R. P. Sijbesma, Angew. Chem. Int. Ed. 2004, 43, 4460, doi: 10.1002/anie.200460040.

[19] A. Piermattei, S. Karthikeyan, R. P. Sijbesma, Nat. Chem. 2009, 1, 133, doi: 10.1038/nchem.167.

[20] S. Karthikeyan, S. L. Potisek, A. Piermattei, R. P. Sijbesma, J. Am. Chem. Soc. 2008, 130, 14968, doi: 10.1021/ja806887k.

[21] P. Michael, W. H. Binder, Angew. Chem. Int. Ed. 2015, 54, 13918, doi: 10.1002/anie.201505678.

[22] D. W. R. Balkenende, S. Coulibaly, S. Balog, Y. C. Simon, G. L. Fiore, C. Weder, J. Am. Chem. Soc. 2014, 136, 10493, doi: 10.1021/ja5051633.

[23] W. Weng, J. B. Beck, A. M. Jamieson, S. J. Rowan, J. Am. Chem. Soc. 2006, 128, 11663, doi: 10.1021/ja063408q.

[24] J. B. Beck, S. J. Rowan, J. Am. Chem. Soc. 2003, 125, 13922, doi: 10.1021/ ja038521k.

[25] P. Chen, Q. Li, S. Grindy, N. Holten-Andersen, J. Am. Chem. Soc. 2015, 137, 11590, doi: 10.1021/jacs.5b07394.

[26] Y. C. Simon, G. L. Fiore, C. Weder, Chimia 2014, 68, 666, doi: 10.2533/ chimia.2014.666.

[27] L. N. Neumann, C. Calvino, Y. C. Simon, S. Schrettl, C. Weder, Dalton Trans. 2018, 47, 14184, doi: 10.1039/c8dt01580e.

[28] a) M. di Giannantonio, M. A. Ayer, E. Verde-Sesto, M. Lattuada, C. Weder, K. M. Fromm, Angew. Chem. Int. Ed. 2018, 57, 11445, doi: 10.1002/ anie.201803524; b) M. Di Giannantonio, M. Ayer, E. Verde-Sesto, M. Lattuada, C. Weder, K. M. Fromm, Chimia 2018, 72, 902.

[29] Y. Sha, Y. Zhang, E. Xu, Z. Wang, T. Zhu, S. L. Craig, C. Tang, ACS Macro Lett. 2018, 7, 1174, doi: 10.1021/acsmacrolett.8b00625.
[30] Y. Sagara, M. Karman, E. Verde-Sesto, K. Matsuo, Y. Kim, N. Tamaoki, C. Weder, J. Am. Chem. Soc. 2018, 140, 1584, doi: 10.1021/jacs.7b12405.

[31] K. Imato, J. C. Natterodt, J. Sapkota, R. Goseki, C. Weder, A. Takahara, H Otsuka, Polym. Chem. 2017, 8, 2115, doi: 10.1039/b203158b.

[32] M. Karman, E. Verde-Sesto, C. Weder, Y. C. Simon, ACS Macro Lett. 2018, 7, 1099, doi: 10.1021/acsmacrolett.8b00591.

[33] C. Kocher, P. Smith, C. Weder, J. Mater. Chem. 2002, 12, 2620, doi: 10.1039/ B202719B.

[34] M. Karman, E. Verde-Sesto, C. Weder, ACS Macro Lett. 2018, 7, 1028, doi: 10.1021/acsmacrolett.8b00520.

[35] Y. Sagara, S. Yamane, M. Mitani, C. Weder, T. Kato, Adv. Mater. 2016, 28, 1073, doi: 10.1002/adma.201502589.

[36] A. P. Haehnel, Y. Sagara, Y. C. Simon, C. Weder, Top. Curr. Chem. 2015, 369, 345, doi: 10.1007/128_2015_640.

[37] C. Löwe, C. Weder, Adv. Mater. 2002, 14, 1625.

[38] B. R. Crenshaw, C. Weder, Chem. Mater. 2003, 15, 4717, doi: 10.1021/ $\mathrm{cm} 034447 \mathrm{t}$.

[39] J. Kunzelman, B. R. Crenshaw, M. Kinami, C. Weder, Macromol. Rapid Commun. 2006, 27, 1981, doi: 10.1002/marc.200600642. [40]

A. Lavrenova, A. Holtz, Y. C. Simon, C. Weder, Macromol. Mater. Eng. 2016, 301, 549, doi: 10.1002/mame.201500403.

[41] C. Calvino, Y. Sagara, V. Buclin, A. P. Haehnel, A. del Prado, C. Aeby, Y. C. Simon, S. Schrettl, C. Weder, Macromol. Rapid Commun. 2018, 9, 40, 1800705, doi: 10.1002/marc.201800705.

[42] A. Lavrenova, D. W. R. Balkenende, Y. Sagara, S. Schrettl, Y. C. Simon, C Weder, J. Am. Chem. Soc. 2017, 139, 4302, doi: 10.1021/jacs.7b00342.

[43] Y. Sagara, T. Mutai, I. Yoshikawa, K. Araki, J. Am. Chem. Soc. 2007, 129, 1520, doi: $10.1021 / \mathrm{ja} 0677362$.

[44] J. Kunzelman, M. Kinami, B. R. Crenshaw, J. D. Protasiewicz, C. Weder, Adv. Mater. 2008, 20, 119, doi: 10.1002/adma.200701772.

[45] Y. Sagara, T. Kato, Nat. Chem. 2009, 1, 605, doi: 10.1038/nchem.411.

[46] Y. Sagara, A. Lavrenova, A. Crochet, Y. C. Simon, K. M. Fromm, C. Weder, Chem. Eur. J. 2016, 22, 4374, doi: 10.1002/chem.201600272.

[47] Y. Sagara, K. Kubo, T. Nakamura, N. Tamaoki, C. Weder, Chem. Mater. 2017, 29, 1273, doi: 10.1021/acs.chemmater.6b04720.

[48] S. T. Zimmermann, D. W. R. Balkenende, A. Lavrenova, C. Weder, J. Brugger, ACS Appl. Mater. Interfaces 2017, 9, 41454, doi: 10.1021/acsami.7b13672.

[49] C. Calvino, C. Weder, Small 2018, 14, 1802489, doi: 10.1002/smll.201802489.

[50] A. P. Esser-Kahn, S. A. Odom, N. R. Sottos, S. R. White, J. S. Moore, Macromolecules 2011, 44, 5539, doi: 10.1021/ma201014n.

[51] J. F. Patrick, M. J. Robb, N. R. Sottos, J. S. Moore, S. R. White, Nature 2016 540, 363, doi: 10.1038/nature21002.

[52] O. Rifaie-Graham, E. A. Apebende, L. K. Bast, N. Bruns, Adv. Mater. 2018 30, 1705483, doi: 10.10002/adma.201705483.

[53] A. Lavrenova, J. Farkas, C. Weder, Y. C. Simon, ACS Appl. Mater. Interfaces 2015, 7, 21828, doi: 10.1021/acsami.5b05797.

[54] C. Calvino, A. Guha, C. Weder, S. Schrettl, Adv. Mater. 2018, 30, 1704603, doi: 10.1002/adma.201704603.

[55] K. M. Herbert, S. Schrettl, S. J. Rowan, C. Weder, Macromolecules 2017, 50, 8845, doi: 10.1021/acs.macromol.7b01607. 